\title{
X-ray diffraction characterization of polymer intercalated graphite oxide
}

\author{
Thomas N. Blanton, ${ }^{\text {a) }}$ and Debasis Majumdar \\ Eastman Kodak Company, Rochester, New York 14650-2106
}

(Received 13 February 2012; accepted 27 February 2012)

\begin{abstract}
Graphite oxide (GO) is generated by treating graphite with strong oxidizers. GO retains the structure of graphite, but does so with a larger and irregular basal plane spacing. The oxidation of graphite results in the formation of epoxide groups, as well as $\mathrm{C}-\mathrm{OH}$ and $\mathrm{COOH}$ groups. It is the presence of some of these moieties that allows GO to be dispersed in water, allowing for its use in waterborne formulations. Although GO does not possess the electrical properties of single-sheet graphene, it can be swelled in water, which allows for intercalation of hydrophilic polymer between GO sheets, resulting in a composite that can be coated to produce a continuous film. After coating it may be possible to chemically convert GO to a reduced graphite oxide (r-GO) with improved electrical conductivity. $\mathrm{X}$-ray diffraction (XRD) is ideally suited to evaluate GO-polymer composite samples for evidence of intercalation or exfoliation of GO. Examples of GO-polymer analysis by XRD are presented, along with results that demonstrate the effect of relative humidity (RH) on neat GO. Knowing the ambient RH during XRD data collection was found to be important to correctly assess the extent of polymer intercalation within the GO lattice. (C) International Centre for Diffraction Data [doi:10.1017/S0885715612000292]
\end{abstract}

Key words: graphite oxide, graphene oxide, intercalation, polymer, X-ray diffraction

\section{INTRODUCTION}

Graphite-based nanoplatelets have garnered considerable attention because of their unique mechanical, thermal and electrical properties (Novoselov et al., 2004). Exfoliated graphite-based platelets when reduced to a single layer of graphene have the potential to revolutionize the flexible electronics market (Geim and Novoselov, 2007). However, the exfoliation process is often cumbersome and has not been reduced to a robust manufacturable practice. Some of the commercially marketed graphene powders or inks are basically micronized graphite, which often does not have the transparency and conductivity required for display applications. In an interesting approach, several groups have focused on creating graphite oxide (GO), sometimes erroneously referred to as graphene oxide, which is relatively easy to exfoliate in aqueous media and can then be subsequently reduced by chemical means or heat treatment. This approach is attractive, since the process chemistry for creating GO in large quantities is well established and GO is easily dispersed in water (Dreyer et al., 2010). Various methods of oxidation of graphite can be found in the literature and the chemical structures created by these methods may vary (Dreyer et al., 2010). A commonly used oxidation method is based on Hummers method (Hummers and Offeman, 1958) or modifications thereof. A proposed molecular structure for GO is based on the LerfKlinowski model (Lerf et al., 1998). As per this model, the GO structure includes regions with unoxidized benzene rings and regions with aliphatic six-membered rings; the oxygen-containing species include epoxides (1,2-ether) and $\mathrm{C}-\mathrm{OH}$ groups with a sprinkling of $\mathrm{COOH}$ groups at the edges. Reduction of GO creates a partially reduced version of graphite oxide (r-GO) which is significantly more conductive than GO (Gilje et al., 2007; Yang et al., 2009). Nanocomposites of GO-polymer have also been studied

\footnotetext{
a) Electronic mail: thomas.blanton@kodak.com
}

(Tung et al., 2011; Vuluga et al., 2011). The hydrophilic nature of GO and its layered structure make it a good host for water-soluble or water-dispersible polymers to intercalate inside the GO lattice.

$\mathrm{XRD}$ is an important analytical technique for the characterization of intercalation and exfoliation in composites comprising polymer and layered materials (Blanton et al, 2000). XRD can accurately measure the interlayer or basal plane $d$-spacing, for example, of GO and monitor intercalation of any species in the gallery of the GO lattice. Whereas the interlayer spacing of graphite is $3.35 \AA$, conversion to GO results in an increase in this basal plane spacing due to functionalization of graphite with oxygen-containing groups (Kou et al., 2010). To determine whether intercalation or exfoliation due to polymers in the GO lattice has occurred in GO-polymer composites, a baseline $d(001)$ basal plane spacing for neat $\mathrm{GO}$ is required. A literature review of XRD results for the analysis of GO reveals a range of $d(001)$ values. Selected publications indicate that this range is from $d(001)=7.8$ to $12 \AA$ (Liu et al., 2002; Bissessur et al., 2006; Stankovich et al., 2007; Cote et al., 2009; Kou et al., 2010; Zhao et al., 2010). In an extreme drying study, Jeong et al. (2009) annealed GO at $200{ }^{\circ} \mathrm{C}$ for $2 \mathrm{~h}$ resulting in $d(001)=6.2 \AA$. However, further annealing resulted in degradation of GO, indicating that thermal processing at this temperature is chemically altering GO. In a modeling study (Boukhvalov and Katsnelson, 2008) of GO with $25 \%$ coverage by hydroxyl groups, the interlayer distance was found to be about $7 \AA$. Since a study by Hofmann et al. in 1932 (Hofmann et al., 1932), it is known that GO can be intercalated by water. The range of $d(001)$ spacings mentioned earlier suggests that XRD data were collected with varying amounts of water intercalated in between GO layers, likely the result of data collection at different relative humidity, RH. In this study, the effect of RH on the GO interlayer spacing is evaluated. Upon establishing baseline parameters, changes in the 
GO interlayer spacing due to interaction of $\mathrm{GO}$ in a polymer matrix can be determined.

\section{EXPERIMENTAL}

\section{A. Sample preparation}

A specimen of $0.5 \mathrm{wt} \%$ GO dispersed in water (N002PS) was obtained from Angstron Materials. Neat GO coatings were formed by depositing a few drops of GO solution onto Teflon or glass microscope slides followed by drying in ambient air. Solutions coated on Teflon could be removed after drying, resulting in self-supporting films. Solutions coated on glass were not removed after drying. Aqueous GO-polymer composite solutions were made by adding GO solutions to $0.5 \mathrm{wt} \%$ polymer solutions to obtain the desired final weight ratio of GO to polymer. Films were prepared on glass microscope slides using the same method as described for neat GO coatings.

\section{B. X-ray diffraction (XRD)}

XRD data were collected using a Rigaku D2000 diffractometer, equipped with a copper rotating anode, a diffractedbeam monochromator tuned to $\mathrm{CuKa}$ radiation and a scintillation detector. Coated films were analyzed in ambient air or under controlled humidity conditions using an environmental cell with a nitrogen-water bubbler. The RH inside the environmental cell was monitored using a VWR digital hygrometer.

\section{RESULTS AND DISCUSSION}

Reflection and transmission mode XRD patterns collected at $30 \% \mathrm{RH}$ for a self-supporting film of GO are shown in Figure 1. In reflection mode, the XRD pattern shows a strong (001) peak, indicating preferred orientation of graphene oxide basal planes parallel to the sample plane. The (001) $d$-spacing is $9.1 \AA$. Based on the (001) peak width, the crystallite size was determined using the Scherrer technique and was found

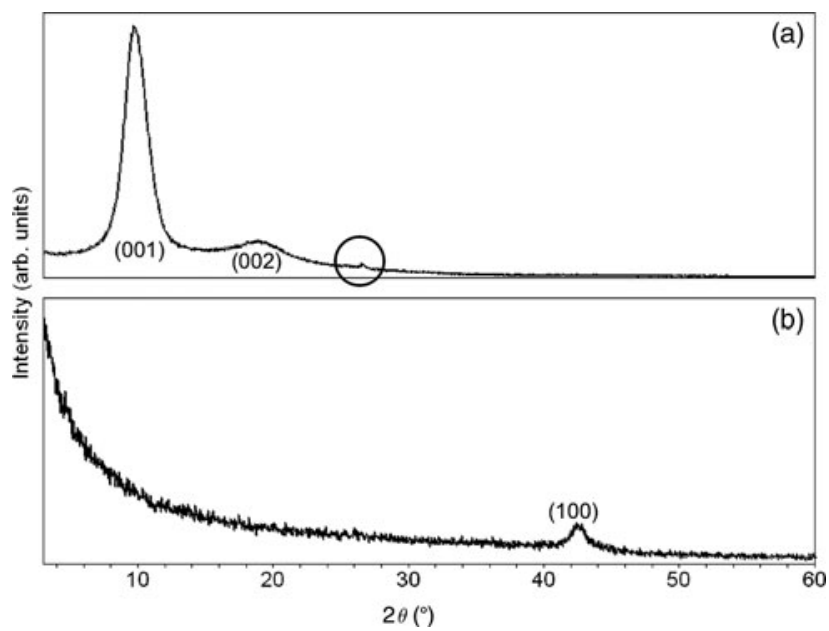

Figure 1. XRD patterns for a self-supporting GO film: (a) reflection mode geometry and (b) transmission mode geometry. Data collected at 30\% RH. Graphite impurity peak indicated by circle in reflection mode XRD pattern. to be $41 \AA$ in the [001]-direction. All films coated with commercial GO solutions were found to have a small amount of graphite, observed in Figure 1(a). In transmission mode, the XRD pattern shows a weak (100) peak, indicating preferred orientation of (100) planes perpendicular to the sample plane. The (100) $d$-spacing is $2.12 \AA$. Based on the (100) peak width, the crystallite size was determined using the Scherrer technique and was found to be $71 \AA$ in the [h00]-direction.

Initial studies of a GO film on glass found that this sample had different (001) $d$-spacings when analyzed on different days. Humidity was believed to be the likely cause. XRD patterns (Figure 2) collected at variable $\mathrm{RH}$ confirmed this hypothesis. The GO $d(001)$ spacing increases (smaller $2 \theta$ ) with increasing humidity, due to water insertion between (001) planes. Note that the (001) GO peak intensity is observed to increase as more water intercalates the GO lattice. The (001) graphite diffraction peak, due to residual graphite in commercial GO solutions, serves as an internal standard for measuring $\mathrm{GO} d(001)$ spacing. A detailed evaluation of the effect of $\mathrm{RH}$ on the GO basal plane spacing was performed using a GO on glass sample in the RH-controlled environmental cell. Diffraction patterns were collected at a specified $\mathrm{RH}$ and $d$ (001) spacings determined (Figure 3 ). From these data, it is apparent that the range of reported $d(001)$ spacings for neat $\mathrm{GO}$ in the literature can be attributed to the $\mathrm{RH}$ during data collection, demonstrating the importance of documenting (and reporting) $\mathrm{RH}$ when presenting XRD results for GO materials.

Having established RH vs. $d(001)$ for GO films, attention is now placed on GO-polymer composites. In Figure 4, XRD patterns for GO-polyethylene oxide (PEO) films on glass collected at $30 \% \mathrm{RH}$ are shown. Based on the shift in the $d(001)$ diffraction peak to lower $2 \theta$, PEO is observed to intercalate the GO lattice. At $50 \mathrm{wt} \%$ PEO $d(001)=15.2 \AA$ compared to $d(001)=9.0 \AA$ for neat GO at $30 \%$ RH. An increase in wt $\%$ PEO $(70 \mathrm{wt} \%)$ did not result in further intercalation as there was no observed increase in the GO $d(001)$ spacing. With PEO polymer intercalated in the GO lattice, the effect of humidity on this composite was evaluated. In Figure 5,

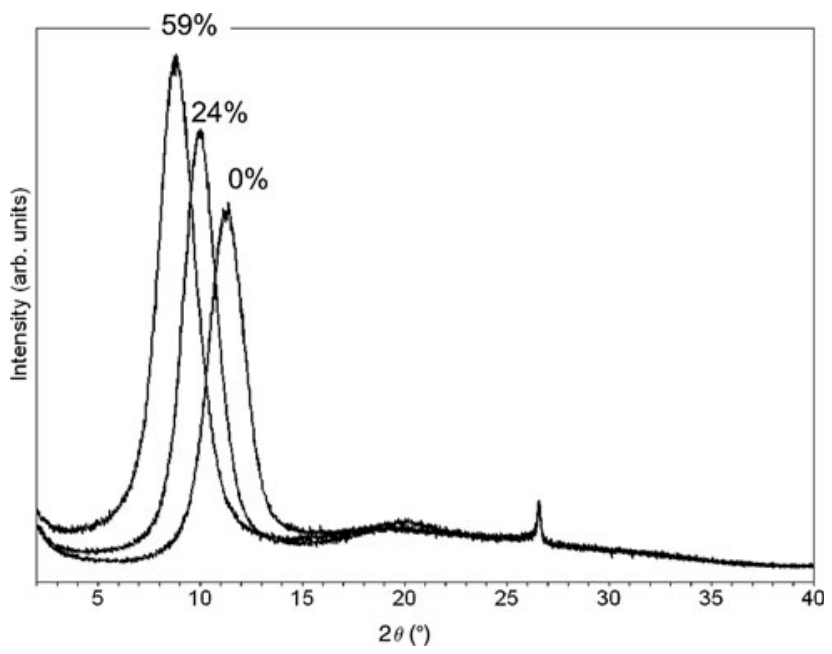

Figure 2. Reflection mode XRD patterns for GO coated on glass, data collected at $0 \%$ (sample in environmental cell purged with dry $\mathrm{N}_{2}$ ), $24 \%$ (sample in lab ambient during winter season) and 59\% (sample in lab ambient after recent rain storm) $\mathrm{RH}$. Graphite (001) peak at $26.62^{\circ} 2 \theta$. 


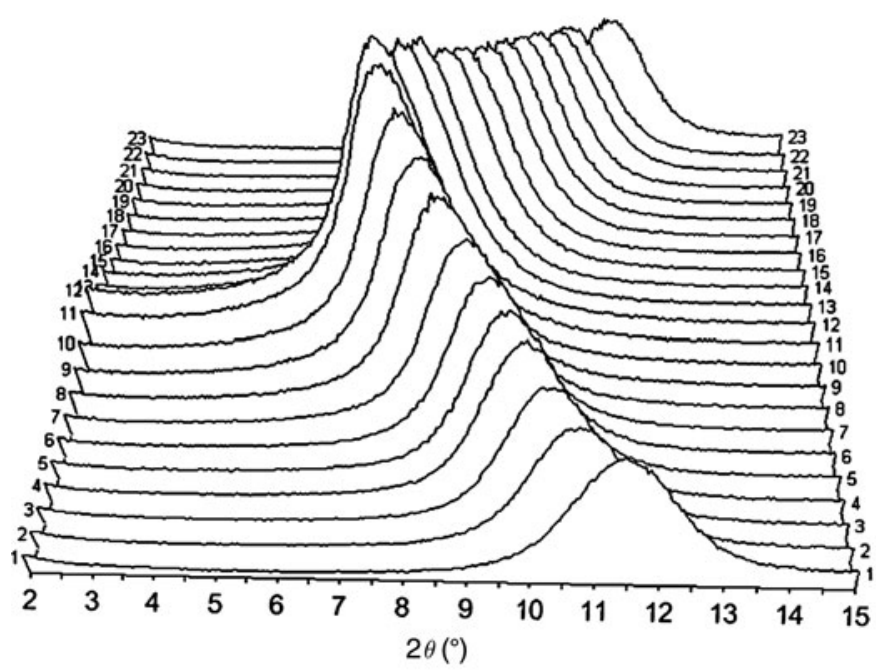

the $d(001)$ spacing changes from $15.5 \AA$ at $50 \%$ RH to $14.7 \AA$ at 0\% RH for the GO:PEO 50:50 composite film coated on glass. Although this change is smaller than what was observed in neat GO, it is a reminder that the RH must be noted when collecting XRD data for GO or GO-polymer samples.

In Figure 6, XRD patterns for GO-polyvinyl pyrrolidone (PVP) films on glass collected at 35\% RH are shown. Similar to what was observed with GO-PEO, PVP is observed to intercalate the GO lattice at $50 \mathrm{wt} \% \mathrm{PVP}$ with the $d(001)=$ $20.0 \AA$, at $70 \mathrm{wt} \%$ PVP $d(001)=31.5 \AA$ and at $90 \mathrm{wt} \%$ PVP it appears GO is exfoliated based on the absence of any $d(001)$ diffraction peak. Before defining a GO composite sample as exfoliated, further analysis should be considered. The GO-PVP XRD patterns in Figure 6 all start at $2^{\circ} 2 \theta$ (44 $\AA$ using $\mathrm{Cu} K \alpha$ ). It is possible to have layered material composites with $d(001)>44 \AA$ (Majumdar et al., 2009). Therefore diffraction patterns collected at lower $2 \theta$ are

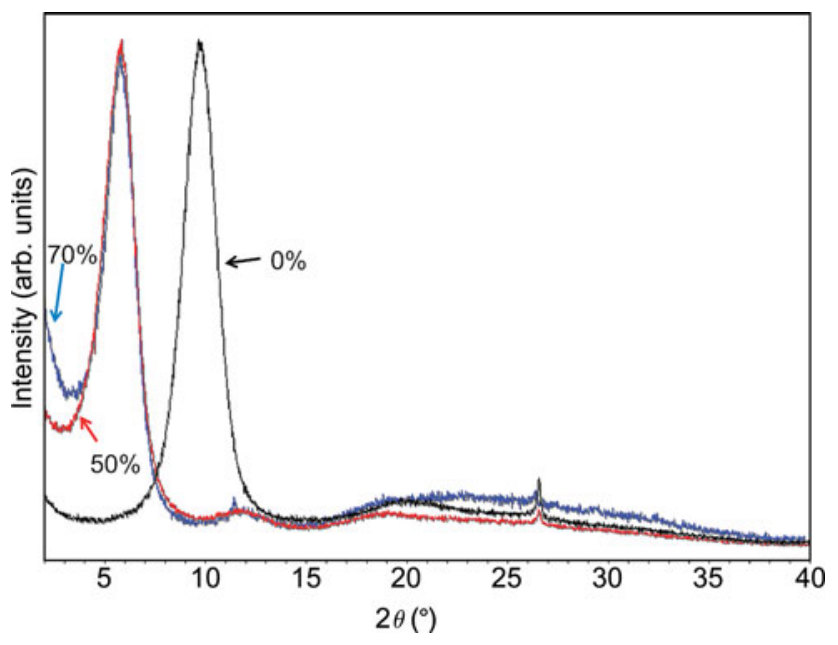

Figure 4. (Color online) Reflection mode XRD patterns for GO-PEO coated on glass, data collected at $30 \%$ RH. Weight percent levels of PEO are 0,50 and $70 \mathrm{wt} \%$, noted on the plot. Graphite (001) peak at $26.62^{\circ} 2 \theta$ (normalized intensities).

\begin{tabular}{|c|c|c|}
\hline Scan \# & \%RH & $\begin{array}{c}\text {-spacing } \\
(\hat{A})\end{array}$ \\
\hline 1 & 0.00 & 7.756 \\
\hline 2 & 5.66 & 8.215 \\
\hline 3 & 12.15 & 8.539 \\
\hline 4 & 22.49 & 8.855 \\
\hline 5 & 30.69 & 9.079 \\
\hline 6 & 39.91 & 9.361 \\
\hline 7 & 49.98 & 9.721 \\
\hline 8 & 60.70 & 10.165 \\
\hline 9 & 69.74 & 10.582 \\
\hline 10 & 79.18 & 10.959 \\
\hline 11 & 90.17 & 11.405 \\
\hline 12 & 93.79 & 11.527 \\
\hline 13 & 85.58 & 11.034 \\
\hline 14 & 79.53 & 10.758 \\
\hline 15 & 68.56 & 10.262 \\
\hline 16 & 60.10 & 9.946 \\
\hline 17 & 49.43 & 9.549 \\
\hline 18 & 39.56 & 9.261 \\
\hline 19 & 29.70 & 8.966 \\
\hline 20 & 19.65 & 8.723 \\
\hline 21 & 10.60 & 8.417 \\
\hline 22 & 6.50 & 8.224 \\
\hline 23 & 0.00 & 7.705 \\
\hline
\end{tabular}

Figure 3. In situ variable humidity XRD patterns for GO coated on glass (reflection mode geometry). The sample was equilibrated $15 \mathrm{~min}$ in an environmental cell at a specified RH before XRD data collection.

required for the instrument conditions used in this study to observe larger $d(001)$ spacings. Low-angle XRD patterns for GO-PVP at $90 \mathrm{wt} \%$ PVP are shown in Figure 7. The initial XRD pattern from Figure 6 started at $2^{\circ} 2 \theta$ with no $(001)$ GO-PVP diffraction peak observed. A scan starting at $1^{\circ} 2 \theta$ using the same diffractometer conditions including slits does show evidence of a diffraction peak; however, significant scatter from the direct beam is present. Using smaller divergence, scatter and receiving slits allows for collection of XRD data started at $1^{\circ} 2 \theta$ that shows a resolved $d(001)$ GO-PVP $90 \mathrm{wt}$ $\%$ diffraction peak with $d(001)=53.2 \AA$ indicating the GO-PVP composite in this case is intercalated, not exfoliated. Another option to improve observation of large $(d(001)$ $d$-spacing data would be to change the X-ray source to a longer wavelength, though attenuation of intensity by air needs to be considered. In the situation where collecting low-angle diffraction data using optimized diffractometer conditions still results in the absence of a $d(001)$ diffraction peak, conclusive

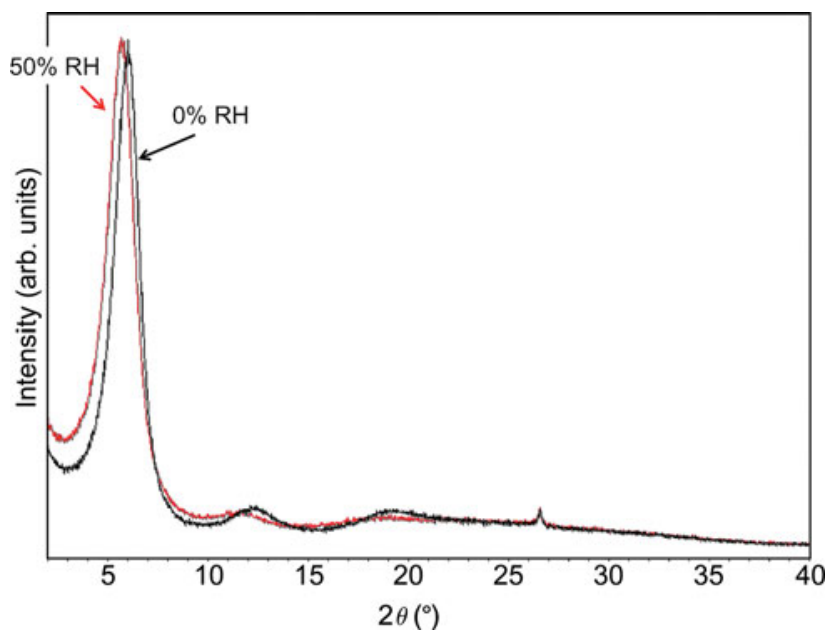

Figure 5. (Color online) Reflection mode XRD patterns for GO-PEO, $50 \mathrm{wt}$ $\%$ each, coated on glass, data collected at 0 and $50 \% \mathrm{RH}$, noted on the plot. Graphite (001) peak at $26.62^{\circ} 2 \theta$ (normalized intensities). 


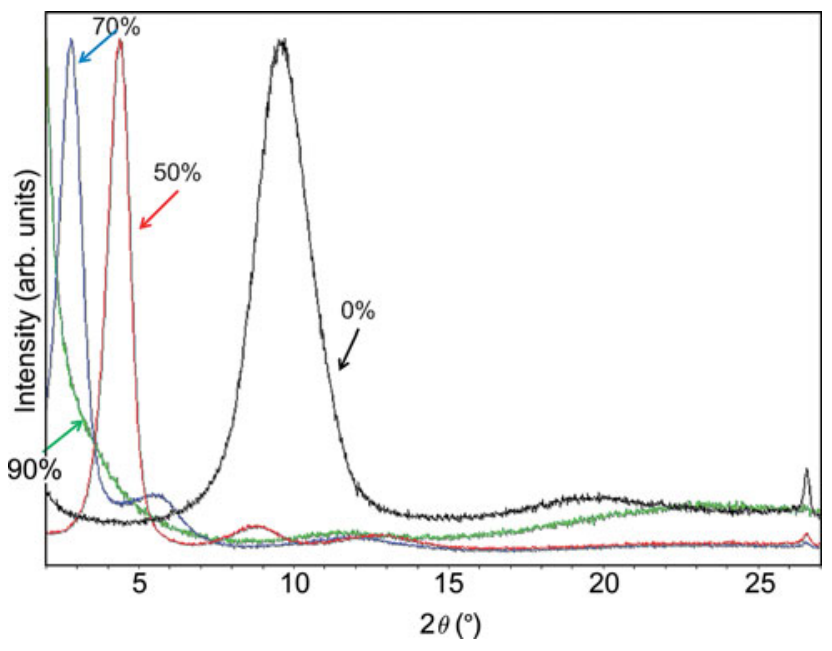

Figure 6. (Color online) Reflection mode XRD patterns for GO-PVP coated on glass, data collected at $35 \%$ RH. Weight percent levels of PVP are $0,50,70$ and $90 \mathrm{wt} \%$, noted on the plot. Graphite (001) peak at $26.62^{\circ} 2 \theta$ (normalized intensities).

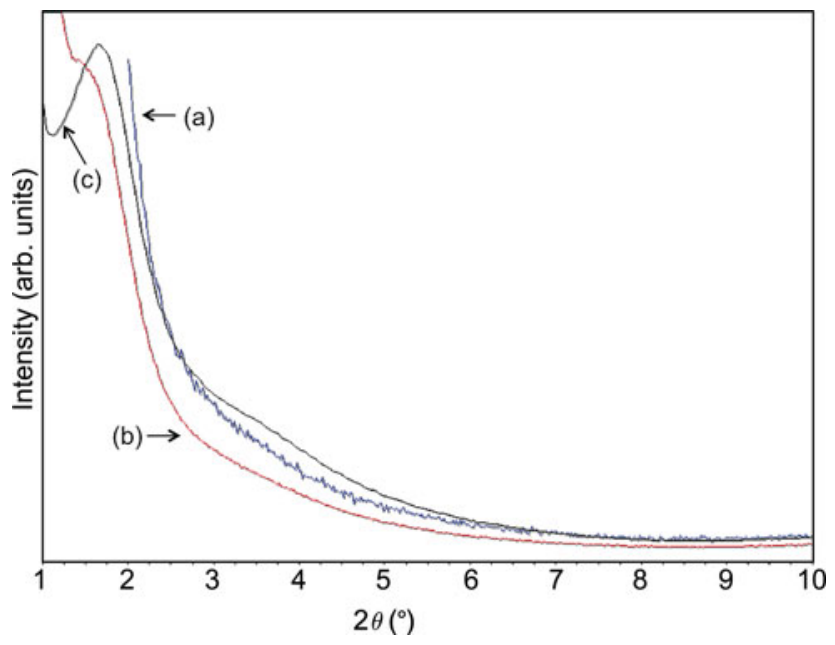

Figure 7. (Color online) Reflection mode XRD patterns for GO-PVP, $90 \mathrm{wt}$ $\%$ PVP, data collected at 35\% RH: (a) the initial XRD scan from Figure 6 started at $2^{\circ} 2 \theta, 1 / 2^{\circ}$ divergence slit, $1 / 2^{\circ}$ scatter slit, $0.6 \mathrm{~mm}$ receiving slit. (b) A scan starting at $1^{\circ} 2 \theta$ using the instrument conditions as 7(a). (c) A scan started at $1^{\circ} 2 \theta, 1 / 4^{\circ}$ divergence slit, $1 / 4^{\circ}$ scatter slit and $0.15-\mathrm{mm}$ receiving slit (normalized intensity).

evidence of exfoliation in layered material composites can be achieved using transmission electron microscopy (TEM) to examine cross-sections of the composite film.

\section{SUMMARY}

- Graphene oxide, GO, has a hexagonal lattice with $a=2.12 \AA$ and $c$ variable depending on ambient humidity.

- Since $d(001)$ varies with humidity, the \%RH during XRD data collection must be recorded to accurately assess any real changes in the GO basal plane spacing. Collection of data at $0 \% \mathrm{RH}$ would be the easiest experimental condition to replicate.

- GO can be intercalated with water-soluble polymers such as PEO and PVP.
- The amount of intercalation, based on the GO-polymer $d(001)$ spacing, is dependent on polymer type and the amount of polymer present, and to a lesser extent the \% RH during data collection.

- Low angle XRD patterns should be collected when GO-polymer composites appear exfoliated based on typical XRD data collection conditions. Further evidence of exfoliation should be confirmed using TEM.

Bissessur, R., Liu, P., and Scully, S. F. (2006). "Intercalation of polypyrrole into graphite oxide," Syn. Metals 156, 1023-1027.

Blanton, T. N., Majumdar, D., and Melpolder, S. M. (2000). "Microstructure of clay-polymer composites," Adv. X-ray Anal. 42, 562-568.

Boukhvalov, D. W., and Katsnelson, M. I. (2008). "Modeling of graphite oxide," J. Am. Chem. Soc. 130, 10697-10701.

Cote, L. J., Cruz-Silva, R., and Huang, J. (2009). "Flash reduction and patterning of graphite oxide and its polymer composite," J. Amer. Chem. Soc. 131, 11027-11032.

Dreyer, D. R., Park, S., Bielawski, C. W., and Ruoff, R. S. (2010). "The chemistry of graphene oxide," Chem. Soc. Rev. 39, 228-240.

Geim, A. K., and Novoselov, K. S. (2007). "The rise of graphene," Nature Mater. 6, 183-191.

Gilje, S., Han, S., Wang, M., Wang, K. L., and Kaner, R. B. (2007). "A chemical route to graphene for device applications," Nano. Lett. 7, 3394-3398.

Hofmann, U., Frenzel, A., Wilm, A., and Csalan, E. (1932). "Eindimensional quelling von graphitsauere und graphit (Die reaktionsweisen des graphits)," Kolloid Z. 61, 297-304.

Hummers, W. S., and Offeman, R. E. (1958). "Preparation of graphitic oxide," J. Am. Chem. Soc. 80, 1339.

Jeong, H. K., Lee, Y. P., Jin, M. H., Kim, E. S., Bae, J. J., and Lee, Y. H. (2009). "Thermal stability of graphite oxide," Chem. Phys. Lett. 470, 255-258.

Kou, L., He, H., and Gao, C. (2010). "Click chemistry approach to functionalize two-dimensional macromolecules of graphene oxide nanosheets," Nanomicro Lett. 2, 177-183.

Lerf, A., He, H., Forster, M., and Klinowski, J. (1998). "Structure of Graphite Oxide Revisited," J. Phys. Chem. B 102, 4477-4482.

Liu, Z. H., Wang, Z. M., Yang, X., and Ooi, K. (2002). "Intercalation of organic ammonium ions into layered graphite oxide," Langmuir 18, $4926-4932$.

Majumdar, D., Blanton, T. N., and Dontula, N. (2009). "Application of Nanocomposites in imaging and display," Chapter 15 in Recent Advances in Polymer Nanocomposites, edited by S. Thomas, G. E. Zaikov and S. V. Valsaraj (VSP Books, Leiden, The Netherlands), pp. $483-526$.

Novoselov, K. S., Geim, A. K., Morozov, S. V., Jiang, D., Zhang, Y., Dubonos, S. V., Grigorieva, I. V., and Firsov, A. A. (2004). "Electric field effect in atomically thin carbon films," Science 306, 666-669.

Stankovich, S., Dikin, A., Piner, R. D., Kohlhass, K. A., Kleinhammes, A., Jia, Y., Wu, Y., Nguyen, S. T., and Ruoff, R. S. (2007). "Synthesis of graphene-based nanosheets via chemical reduction of exfoliated graphite oxide," Carbon 45, 1558-1565.

Tung, V. C., Kim, J., Cote, L. J., and Huang, J. (2011). "Sticky interconnects for solution-processed tandem solar cells," J. Am. Chem. Soc. 133, 9262 9265.

Vuluga, D., Thomassin, J., Molenberg, I., Huynen, I., Gilbert, B., Jerome, C., Alexandre, M., and Detrembleur, C. (2011). "Straightforward synthesis of conductive graphene/polymer nanocomposites from graphite oxide," Chem. Commun. 47, 2544-2546.

Yang, D., Velamakanni, A., Bozoklu, G., Park, S., Stoller, M., Piner, R. D., Stankovich, S., Jung, I., Field, D. A., Ventrice, C. A., and Ruoff, R. S. (2009). "Chemical analysis of graphene oxide films after heat and chemical treatments by x-ray photoelectron and micro-Raman spectroscopy," Carbon 47, 145-152.

Zhao, X., Zhang, Q., Hao, Y., Li, Y., Fang, Y., and Chen, D. (2010). "Alternate multilayer films of poly(vinyl alcohol) and exfoliated graphene oxide fabricated via a facial layer-by-layer assembly," Macromol. 43, 9411-9416. 